\title{
IgA Nephropathy Presenting as Reno-pulmonary Syndrome
}

\author{
10.5005/jp-journals-10028-1455 \\ CPC editor \\ Pathology discussant \\ Pathology resident \\ Clinical discussant \\ CPC chairperson \\ Date of CPC
}

\section{Case Description}

A 23-year-old male patient presented with a complaint of fever and cough for 10 days and shortness of breath for 2 days. Fever was of high grade and associated with chills and rigors. It reached a maximum temperature of up to $104^{\circ} \mathrm{F}$ with evening rise and was relieved with oral medication. Cough was insidious in onset, with scanty mucoid expectoration. It was non-paroxysmal, without any diurnal or postural variation. There was no history of hemoptysis. Dyspnea was gradual in onset and rapidly progressed to dyspnea at rest with orthopnea. No history of chest pain, rhinorrhea, wheeze, headache, focal neurological deficit, bleeding manifestations, joint pain, myalgias, dysuria, hematuria, or decrease in urine output. No history of diabetes, pulmonary tuberculosis, epilepsy, or asthma.

\section{Details of Previous Admission}

Three months before the current admission (March 2013) patient presented with a complaint of swelling of feet, facial puffiness, and frothuria for 1 month. On evaluation, his serum creatinine was 3.1 to $4 \mathrm{mg} \%$. Urine routine examination showed albumin $4+$, urine microscopy showed full-field RBCs, 3 to $5 \mathrm{WBC} / \mathrm{HPF}$, 24-hour urine protein 1,219 g. ANA-Negative, ANCA-Negative. He was clinically diagnosed with nephritic-nephrotic syndrome. A kidney biopsy was done and reported as IgA nephropathy with moderate IFTA (M1E1S1TI). Patient received methylprednisolone pulse for 3 days followed by oral steroids and cyclophosphamide.

\section{EXAMINATION}

The patient was conscious and oriented. He was dyspneic at rest with an accessory muscle of respiration working and had pallor. Chest: Bilateral extensive crepitus was present.

$\mathrm{SpO}_{2}-74 \%$ at room air and $86 \%$ on $\mathrm{FiO}_{2} 0.5$ with a venti mask. Initial ABG showed respiratory alkalosis with hypoxia. BP-110/80, PR-120/minute regular, RR-36/minute. No icterus, cyanosis, clubbing, or pedal edema. JVP was not raised.

\section{Investigations (Tables 1 to 5 ) Radiological Investigations}

- Serial chest radiographs and HRCT lungs were done

\section{Radiological Differential Diagnosis}

- Infective: Polymicrobial, Organizing pneumonia

- Bacterial: Mycoplasma, Tuberculosis

- Fungal: Pneumocystis jirovecii, Aspergillus, Mucor

- Viral: CMV, adenovirus

\begin{abstract}
${ }^{1-3}$ Department of Histopathology, Postgraduate Institute of Medical Education and Research, Chandigarh, India

${ }^{4}$ Department of Nephrology, Postgraduate Institute of Medical Education and Research, Chandigarh, India

${ }^{5}$ Department of Internal Medicine, Postgraduate Institute of Medical Education and Research, Chandigarh, India
\end{abstract}

Corresponding Author: Ritambhra Nada, Department of Histopathology, Postgraduate Institute of Medical Education and Research, Chandigarh, India, Phone: +91 7087008143, e-mail: ritamduseja@yahoo.com

How to cite this article: Nada R, Parwaiz A, Gupta $\mathrm{KL}$, et al. IgA Nephropathy Presenting as Reno-pulmonary Syndrome. J Postgrad Med Edu Res 2021;55(4):197-204.

Source of support: Nil

Conflict of interest: None

- Acute interstitial pneumonia

- Diffuse alveolar hemorrhage

USG Abdomen-6-6-2013

- Mild hydronephrosis in the right kidney but no collection

- No evidence of DVT in bilateral lower limb

USG Abdomen-9-6-13

- Moderate ascites

- ECG-sinus tachycardia

- S. procalcitonin (8-6-13)-112.9 and (19-6-13) 83.68

- S. galactomannan-(10-6-13)-0.22 and (16-6-13) 0.30

- Blood bacterial culture-Negative

- Fungal serology (8-6-13)-Positive for A. flavus, A. fumigatus, and $A$. niger.

- Blood fungal culture-Negative

Table 1: Endotracheal aspirate-culture and sensitivity

\begin{tabular}{lll}
\hline Date & Results & Drug sensitivity \\
\hline 8-6-13 & Sterile & Klebsiella pneumoniae \\
$11-6-13$ & $\begin{array}{l}\text { Cefotaxime, cefipime, } \\
\text { amikacin, imipenem }\end{array}$ \\
$14-6-13$ & $\begin{array}{l}\text { Klebsiella pneumoniae, } \\
\text { Pseudomonas aeruginosa }\end{array}$ & $\begin{array}{l}\text { Cefotaxime, ceftazidime, } \\
\text { cefipime, amikacin, imipenem }\end{array}$ \\
18-6-13 & Acinetobacter complex & Tobramycin, colistin \\
\hline
\end{tabular}

(-) The Author(s). 2021 Open Access This article is distributed under the terms of the Creative Commons Attribution 4.0International License (https://creativecommons. org/licenses/by-nc/4.0/), which permits unrestricted use, distribution, and non-commercial reproduction in any medium, provided you give appropriate credit to the original author(s) and the source, provide a link to the Creative Commons license, and indicate if changes were made. The Creative Commons Public Domain Dedication waiver (http://creativecommons.org/publicdomain/zero/1.0/) applies to the data made available in this article, unless otherwise stated. 
IgA Nephropathy Presenting as Reno-pulmonary Syndrome

Table 2: Hemogram findings

\begin{tabular}{|c|c|c|c|c|c|c|c|}
\hline & $28-5-13$ & $5-6-13$ & $7-6-13$ & $10-6-13$ & $15-6-13$ & $20-6-13$ & $24-6-13$ \\
\hline $\mathrm{HB}(\mathrm{g} \%)$ & 9.0 & 7.9 & 6.9 & 6.9 & 7.8 & 6.9 & 7.0 \\
\hline $\mathrm{TLC} / \mathrm{mm}^{3}$ & & 14,200 & 13,500 & 22,100 & 30,400 & 28,700 & 22,900 \\
\hline $\begin{array}{l}\text { Differential } \\
\text { P/L/M/E }\end{array}$ & & $95 / 5$ & $90 / 6 / 2 / 2$ & $90 / 7 / 2 / 1$ & $75 / 20 / 2 / 1$ & $89 / 7 / 3 / 1$ & $79 / 16 / 4 / 1$ \\
\hline Plt count $/ \mathrm{mm}^{3}$ & & 210,000 & 190,000 & 201,000 & 244,000 & 140,000 & 131,000 \\
\hline ESR & & & 65 & & & & \\
\hline
\end{tabular}

Table 3: Biochemistry—laboratory values

\begin{tabular}{|c|c|c|c|c|c|c|c|c|}
\hline & $28-5-13$ & $5-6-13$ & $7-6-13$ & $10-6-13$ & $15-6-13$ & $19-6-13$ & $22-6-13$ & $25-6-13$ \\
\hline $\mathrm{Na}+$ & & 140 & 132 & 132 & 138 & 130 & 134 & 123 \\
\hline $\mathrm{K}+$ & & 3.2 & 4.3 & 3.2 & 5.1 & 4.7 & 4.9 & 4.5 \\
\hline Urea & & 198 & 218 & 286.5 & 358 & 321 & 347 & 365 \\
\hline Creat & 2.88 & 4.5 & 5.8 & 7.09 & 4.4 & 3.1 & 3.6 & 4.3 \\
\hline Bil (T/D) & & $0.2^{\prime}$ & 0.4 & 0.2 & 0.3 & & 0.4 & 0.5 \\
\hline SGOT/SGPT/ALP & & & $58 / 14 / 44$ & $16 / 15 / 77$ & $21 / 10 / 120$ & & 29/2/194 & $34 / 8 / 277$ \\
\hline Total protein/ALb & & & $4.3 / 2.3$ & $4.8 / 3.1$ & $6.2 / 3.1$ & & $5.2 / 2.8$ & \\
\hline $\mathrm{Ca} / \mathrm{PO} 4 / \mathrm{Mg}$ & & & 6.8/8.1/1.5 & $9.5 / 13 / 1.9$ & NA/NA/2.35 & & & \\
\hline LDH & & & 2217 & 862 & & & & \\
\hline
\end{tabular}

Table 4: Arterial blood gas value

\begin{tabular}{|c|c|c|c|c|c|c|c|c|c|}
\hline & $6-6-13$ & $8-6-13$ & $11-6-13$ & $15-6-13$ & $18-6-13$ & $21-6-13$ & $23-6-13$ & $25-6-13$ & $25-6-13$ \\
\hline $\mathrm{pH}$ & 7.52 & 7.43 & 7.42 & 7.21 & 7.25 & 7.26 & 7.19 & 7.15 & 7.05 \\
\hline $\mathrm{pO}_{2}$ & 44.7 & 59.7 & 51.5 & 62.6 & 80.3 & 62.6 & 63.4 & 55.8 & 36.9 \\
\hline $\mathrm{PCO}_{2}$ & 29.2 & 33.9 & 25 & 49.8 & 44 & 40.4 & 39.8 & 42.5 & 75.1 \\
\hline $\mathrm{HCO}_{3}{ }^{-}$ & 23.7 & 22.3 & 15.9 & 19.5 & 19 & 18.1 & 14.6 & 14.6 & 20.7 \\
\hline $\mathrm{SpO}_{2}$ & 86.2 & 91.3 & 86.3 & 85 & 92 & 87 & 83.8 & 75.9 & 43.1 \\
\hline $\mathrm{FiO}_{2}$ & & 0.5 & 0.38 & 0.4 & 0.4 & 1 & 0.75 & 1 & 1 \\
\hline
\end{tabular}

Mini BAL

- Positive for Klebsiella pneumoniae ("sensitive" to cefotaxime, ceftazidime, cefipime, amikacin, imipenem).

- Stain for fungus and P. jirovecii-Negative.

- Cytology-Negative for hemosiderin-laden macrophages.

Endotracheal aspirate-Negative for AFB stain and fungal elements

\section{Other Investigations}

- ANA/ANCA-Negative.

- CMV PCR-Negative.

- Malaria antigen negative.

- 24-hour protein-504 mg.

\section{Course and Management}

This 23-year-old man diagnosed to have IgA nephropathy in March 2013, received pulse methylprednisolone, followed by oral steroids and cyclophosphamide $75 \mathrm{mg} /$ day for 5 weeks. Now patient presented with fever, productive cough, and dyspnea. On examination, he had pallor, respiratory distress, bilateral crackles, tachycardia, and poor oxygen saturation of $74 \%$ at room air. Chest X-ray and CT chest showed bilateral consolidation $(\mathrm{R}>\mathrm{L})$. Investigations showed anemia, leukocytosis, coagulopathy, normal platelet count, non-oliguric AKI, and type I respiratory failure. A
Table 5: Coagulation profile

\begin{tabular}{lll}
\hline & $7-6-2013$ & $19-6-2013$ \\
\hline PT (12-15 seconds) & 22 & 15 \\
aPTT (28-35 seconds) & 28 & 26 \\
PTI\% & 59 & 86 \\
INR & 1.68 & 1.15 \\
Fibrinigen $(2-4 \mathrm{~g} / \mathrm{L}) / \mathrm{D}-$ & $8.5 /$ Negative & - \\
dimer $(0.5 \mu \mathrm{g} / \mathrm{mL})$ & & \\
\hline
\end{tabular}

possibility of pneumocystis pneumonia was considered and the patient was started on clindamycin (as he was hypersensitive to cotrimoxazole and doxycycline) and broad-spectrum antibacterial cover with IV piperacillin and tazobactam. As the patient did not improve after 48 hours, a possibility of diffuse alveolar hemorrhage was considered because of a drop in hemoglobin in serial hemograms (9 g\%-6.7 $\mathrm{g} \%$ ) and new-onset bilateral pulmonary infiltrates. The patient was given a session of plasmapheresis (developed an anaphylactic reaction to 4th FFP). At this time Mini BAL was negative for hemosiderin-laden macrophages and $P$. jirovecii. Blood and sputum cultures were sterile and there was no evidence of hemolysis. BAL culture and sensitivity showed pan sensitive Klebsiella and hence the possibility of severe sepsis with MODS related to $K$. pneumoniae was considered and so a combination of piperacillin and tazobactam was continued. Fever 
subsided with reduced $\mathrm{O}_{2}$ requirement. But he started deteriorating again in the form of tachypnea and hypoxemia requiring ventilatory support and high-grade fever. In the absence of any other infective pathology and K. pneumoniae, Pseudomonas aeruginosa grew from endotracheal aspirate which was sensitive to imipenem, antibiotics changed to imipenem for covering these organisms. Following which fever subsided and urine output improved, however, his ventilatory requirement continued to be high. Later, endotracheal aspirate revealed gram-negative bacteria confirmed to be Acinetobacter which was sensitive to colistin. The patient was started on colistin; however, he developed non-fluid responsive hypotension and worsening of ventilatory requirement. Ionotropic support was added for worsening hypotension. Despite all the efforts, he continued to deteriorate. On 26-6-2013, his saturation decreased from 90 to $80 \%$, gradually decreasing up to $30 \%$. The patient sustained bradycardia followed by cardiac arrest from which he could not be revived and was declared dead at 1:40 AM on 26-6-13.

\section{Final Clinical Diagnosis}

- IgA nephropathy-CKD stage IV.

- Pneumonia in an immunocompromised host.

- Ventilator-acquired pneumonia (polymicrobial) with multiorgan failure.
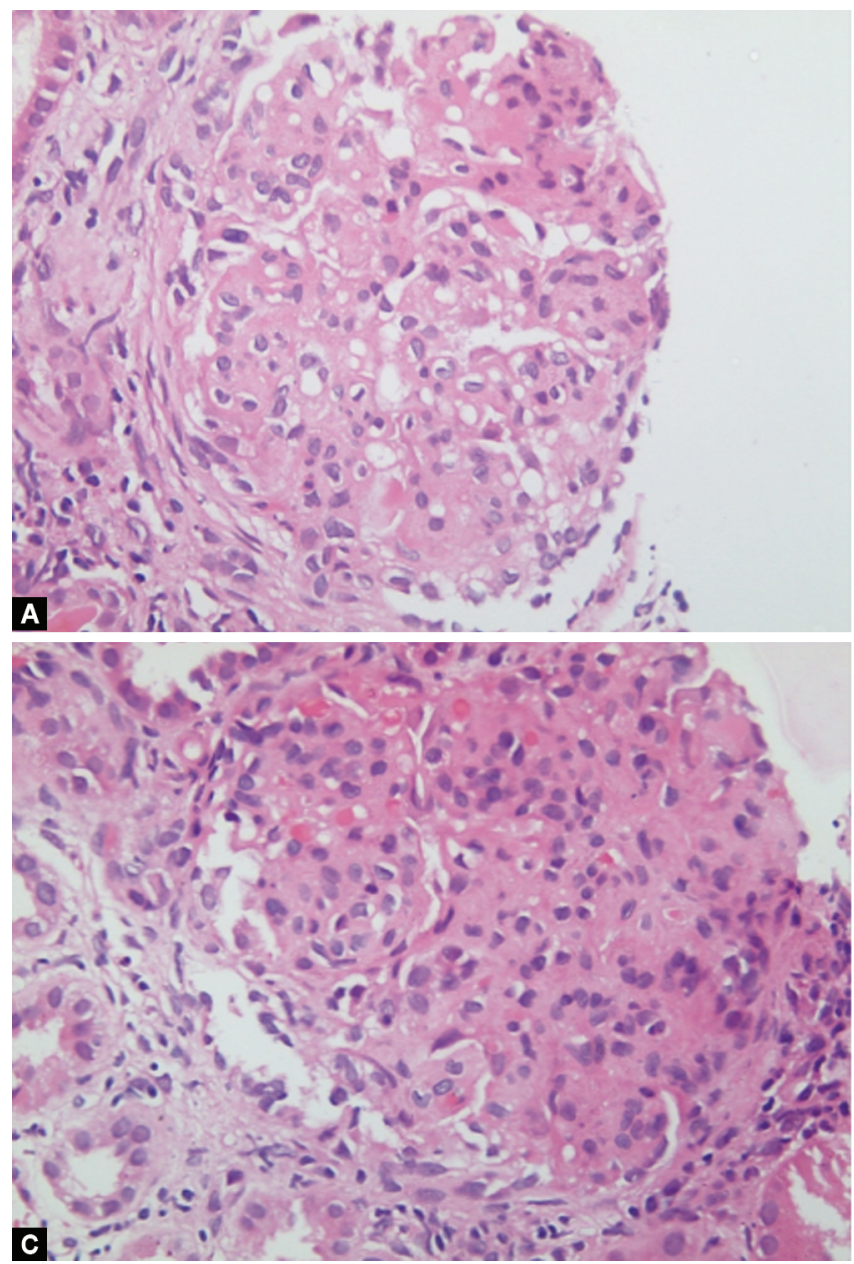

Figs 1 A to D: Photomicrograph of antemortem renal biopsy shows a glomerulus with fibrocellular crescents and diffuse endocapillary proliferation. [(A and C) H\&E stain; (B and D) PAS stain; original magnification 200x]

\section{Cause of Death}

- Refractory hypoxemia

- Refractory shock

\section{Clinical Discussion}

Prof S Varma: Thank you, Dr KL Gupta, for the detailed presentation.

Prof S. Jain: It seems to be a clear-cut case of IgA nephropathy on immunosuppression. There might be evidence of infection in the lungs contributing to pulmonary hemorrhages.

Prof KL. Gupta: I do agree but investigations did not support it.

Dr Manish Rathi: Can there be a role of ANCA-associated pathology contributing to both renal and lung pathology? It is known to coexist.

Prof KL. Gupta: Yes it is possible clinically but ANCA was negative.

Prof S Varma: Since this seems to be a straight forward case let us see pathology.

\section{Pathology Findings}

A partial autopsy was performed on this 23-year-old male patient who is a diagnosed case of IgA nephropathy (Figs 1 to 3). Prosector noted that pleural, peritoneal, and pericardial cavity was within normal limits.
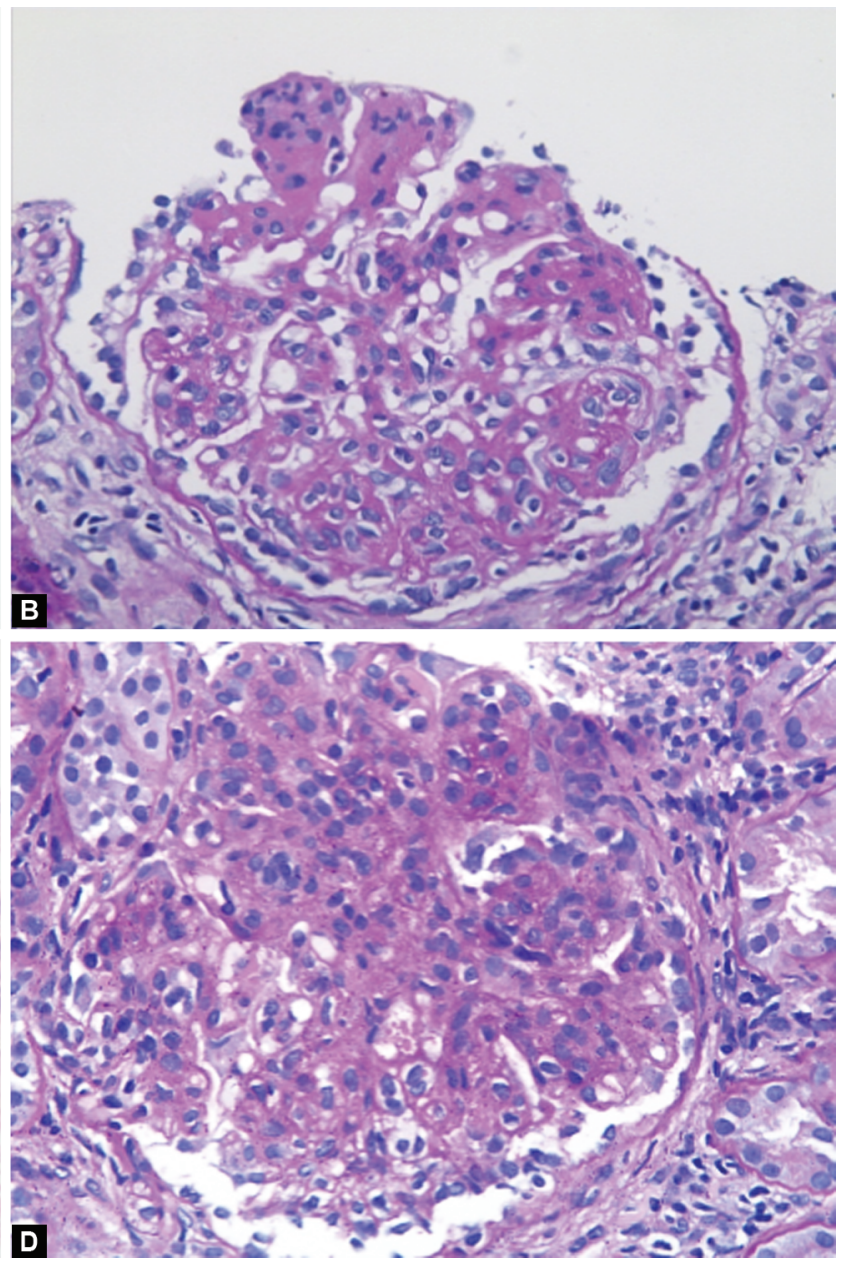

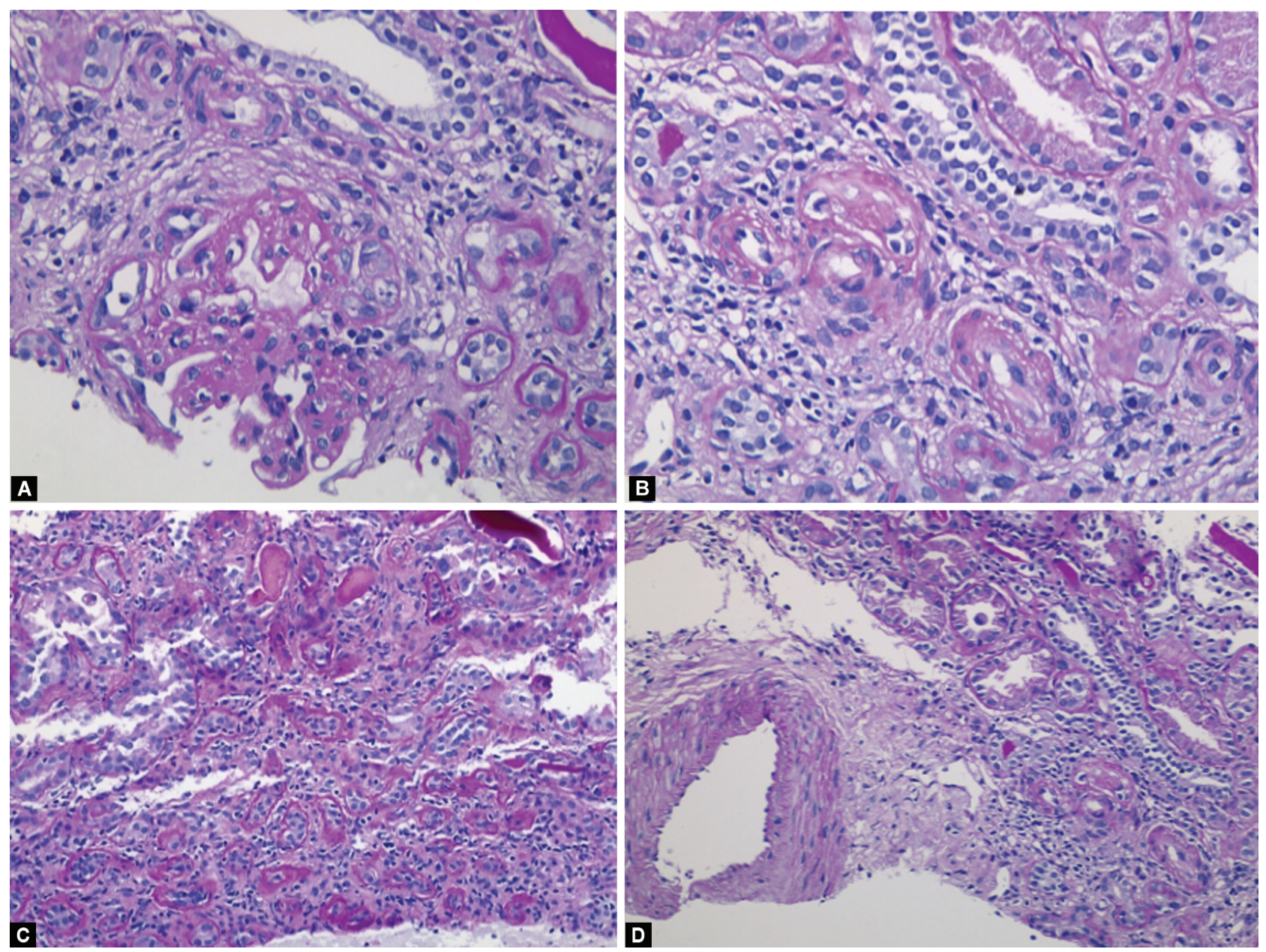

Figs 2A to D: (A) Photomicrograph of antemortem renal biopsy shows a glomerulus with segmental sclerosis; (B) Arterioles with features of subacute thrombotic microangiopathy; (C) Interstitial fibrosis and tubular atrophy with accompanying inflammation; (D) Small renal artery with mild arteriosclerosis [(A) H\&E stain; (B)PAS stain; 200x, (C and D) PAS stain; 100x)

Kidney weighed $220 \mathrm{~g}$ capsular surfaces showed finely granular pinpoint hemorrhages with cortical cysts measuring 1 to $2 \mathrm{~cm}$ (Fig. 4). Microscopy showed a mesangioproliferative pattern of injury with variable degrees of sclerosis and synechiae formation along with fibrocellular, fibrous crescents, and globally sclerosed glomeruli (50\%). There was patchy moderate IFTA with accompanying mild inflammation. Moderate arteriosclerosis with evidence of chronic thrombotic microangiopathy was also noted (Fig. 5). On direct immunofluorescence, the glomeruli show mesangial deposits as well deposits along the capillary wall for IgA $3+, \lg G 1+, \lg M 2+, C 32+$, Kappa 1+, and Lambda 1+. Overall features were of IgA nephropathy with moderate arteriosclerosis (M2E1S3T2 C2).

Lung-weighted $2400 \mathrm{~g}$-lungs were heavy. Pleura was dull and it was subcrepitant to feel. Cut surface showed patchy consolidation with areas of hemorrhages (Fig. 6). Microscopy showed massive areas of pulmonary hemorrhages. It was both fresh and old hemorrhages. Direct immunofluorescence showed IgA and focal IgG deposition along the alveolar septal capillaries and basement membrane (Figs 7 and 8).

There was evidence of acute pneumonia and diffuse alveolar damage. Features of the organization were also noted in the form of interstitial fibrosis and bronchiolitis obliterans. Grams stain did not reveal any gram-positive bacilli. ZN stain for AFB was negative. PAS stain did not highlight any fungal profile (Fig. 9).
Overall features are IgA-mediated pulmonary hemorrhages (fresh and old), acute pneumonia, and diffuse alveolar damage.

Heart weighed $329 \mathrm{~g}$. The external surface was grossly unremarkable. All four chambers and valves were grossly unremarkable. Microscopy did not reveal any pathology.

Liver-weighted $1,500 \mathrm{~g}$. The capsular and cut surface was grossly unremarkable. No focal lesion or nodularity was identified. Microscopy showed maintained lobular architecture with maintained reticulin framework and no increase in fibrosis. The pancreas was grossly as well as microscopically normal.

Spleen weighed $140 \mathrm{~g}$ and showed small inactive lymphoid follicles.

The gastrointestinal tract was grossly and microscopically normal.

Bone marrow showed an adequate representation of all the three hematopoietic elements and increased macrophages.

Testis microscopy showed Sertoli cells only. There was no evidence of spermatogenesis.

\section{Final Autopsy Diagnosis}

- IgA nephropathy (M2E1S3T2C 2) with arterionephrosclerosis.

- Diffuse pulmonary hemorrhages-immune complex-mediated.

- Acute pneumonia with bronchiolitis obliterans with organizing pneumonia (BOOP). 

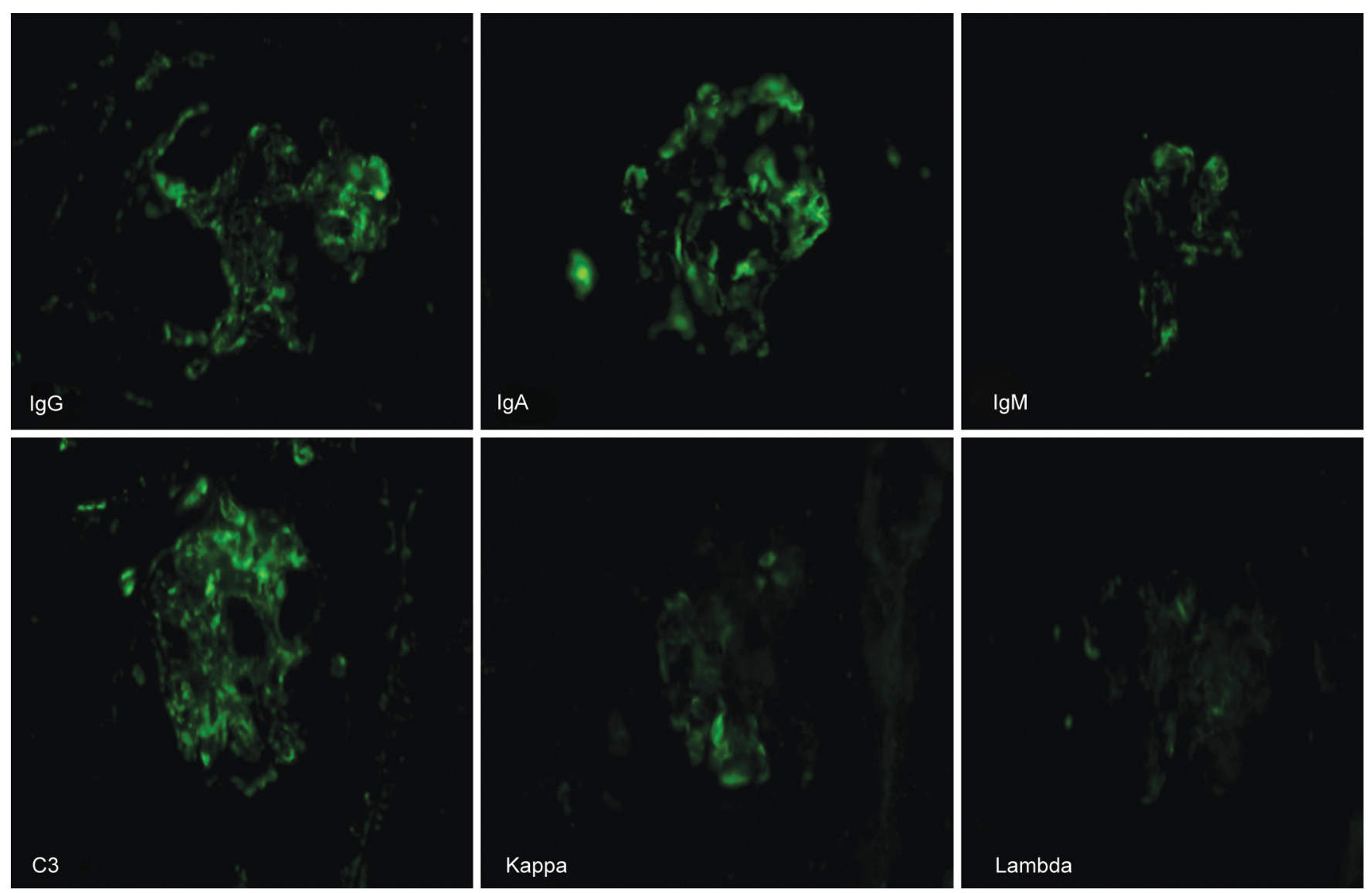

Fig. 3: Composite images of direct immunofluorescence in kidney showing mesangial deposits and focal deposits along the basement membrane of $\lg G 1+, \lg A 2+$, C3 2+, IgM 1+, Kappa 2+, and Lambda 1+ (FITC, 200x)
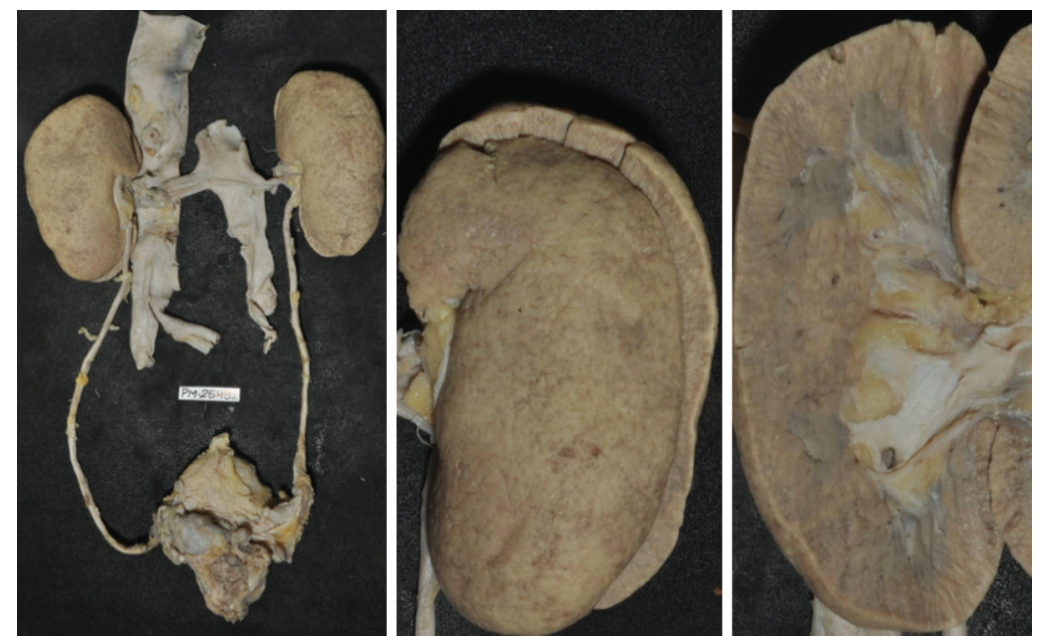

Fig. 4: Gross images: Both the kidneys with capsular surface showing finely granular pinpoint hemorrhages with cortical cysts

- Diffuse alveolar damage.

- Testicular atrophy.

\section{Discussion}

Prof S Varma: Thank you, Dr Ritambhra for the lucid presentation.

It was perfect clinicopathologic correlation today.
Prof S Jain: Can there still be ANCA-associated pathology as we have tested the routine ANCAs.

Dr Ritambhra: Yes, sir quite possible but on pathology, we have no way of proving that. Only the fact no other organ including GIT had any evidence of vasculitis.

Prof S Varma: It was perfect clinicopathologic correlation today. 

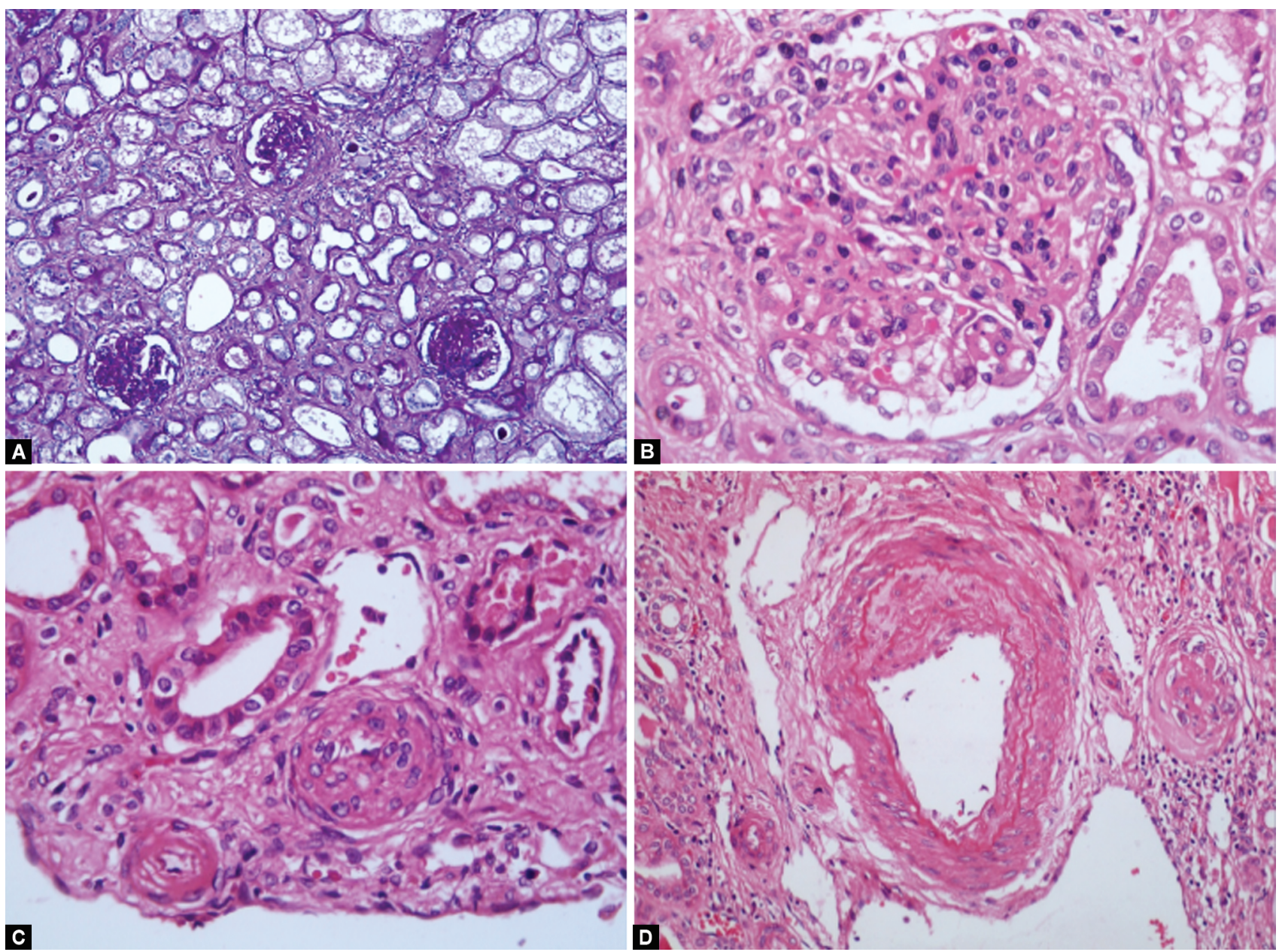

C

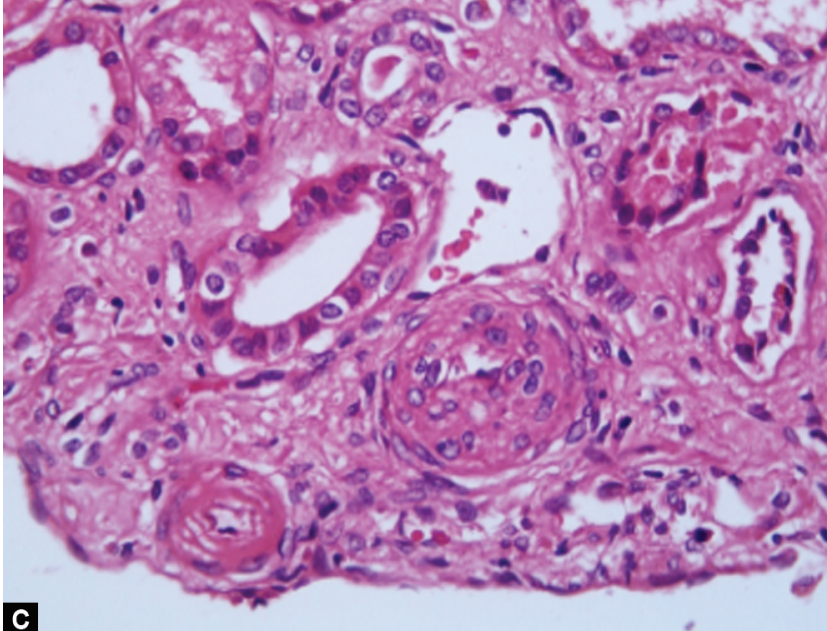

Figs 5A to D: (A and B) Photomicrograph from kidney showing glomeruli with fibrocellular crescents and diffuse endocapillary hypercellularity; (C) Arterioles with features of subacute thrombotic microangiopathy; (D) Small arteries showing moderate arteriosclerosis [(A) PAS stain; 40x, (B to D) H\&E stain; 200x]

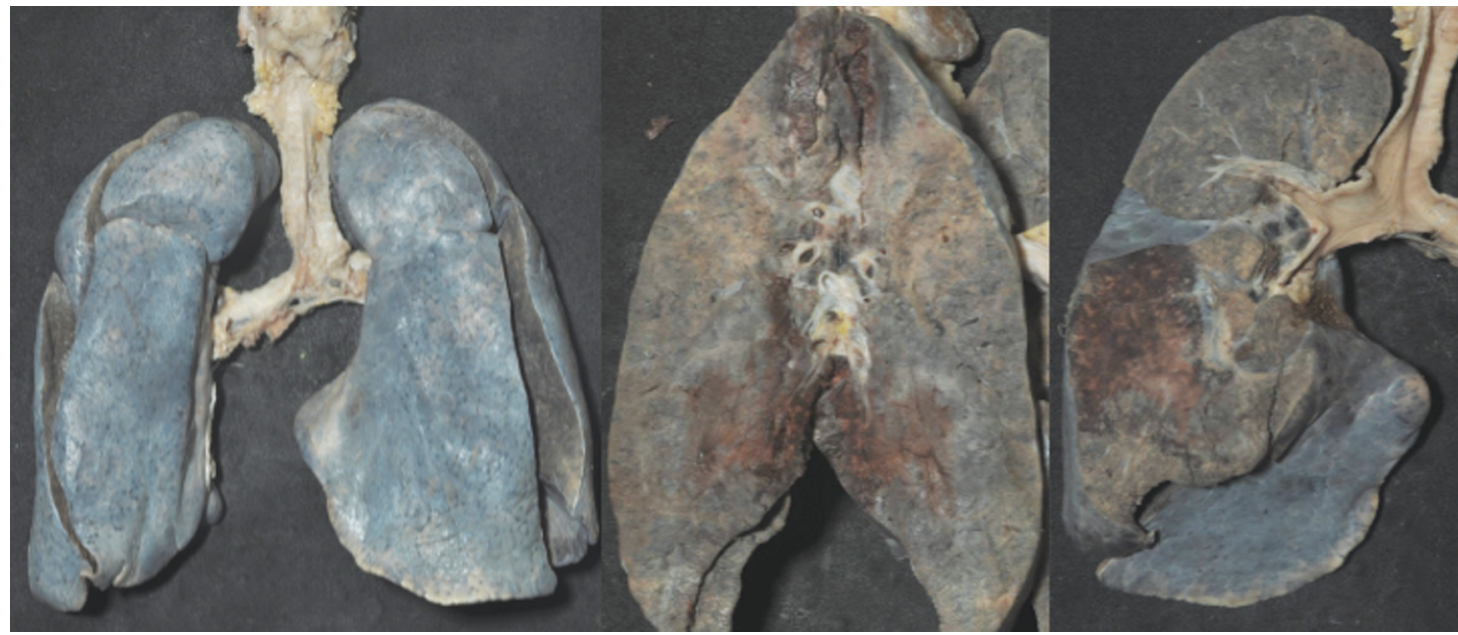

Fig. 6: Gross images: (A) Pleura is dull in appearance; (B and C) Cut surface showing patchy consolidation with areas of hemorrhages

IgA nephropathy is considered the most common primary glomerulonephritis in the world. IgA nephropathy has been associated with other diseases (hepatobiliary, gastrointestinal, rheumatological, and neoplastic) conditions. A pulmonary-renal syndrome is more commonly associated with anti-GBM disease,
ANCA-associated vasculitis, and systemic lupus erythematosus. ${ }^{1}$ Available literature review indicates pulmonary involvement is very rare in IgA nephropathy. In the largest review series of 23 cases of IgA nephropathy and lung involvement, 19 cases showed diffuse alveolar hemorrhage. ${ }^{2}$ Mean age of the patients was $37.4 \pm$ 

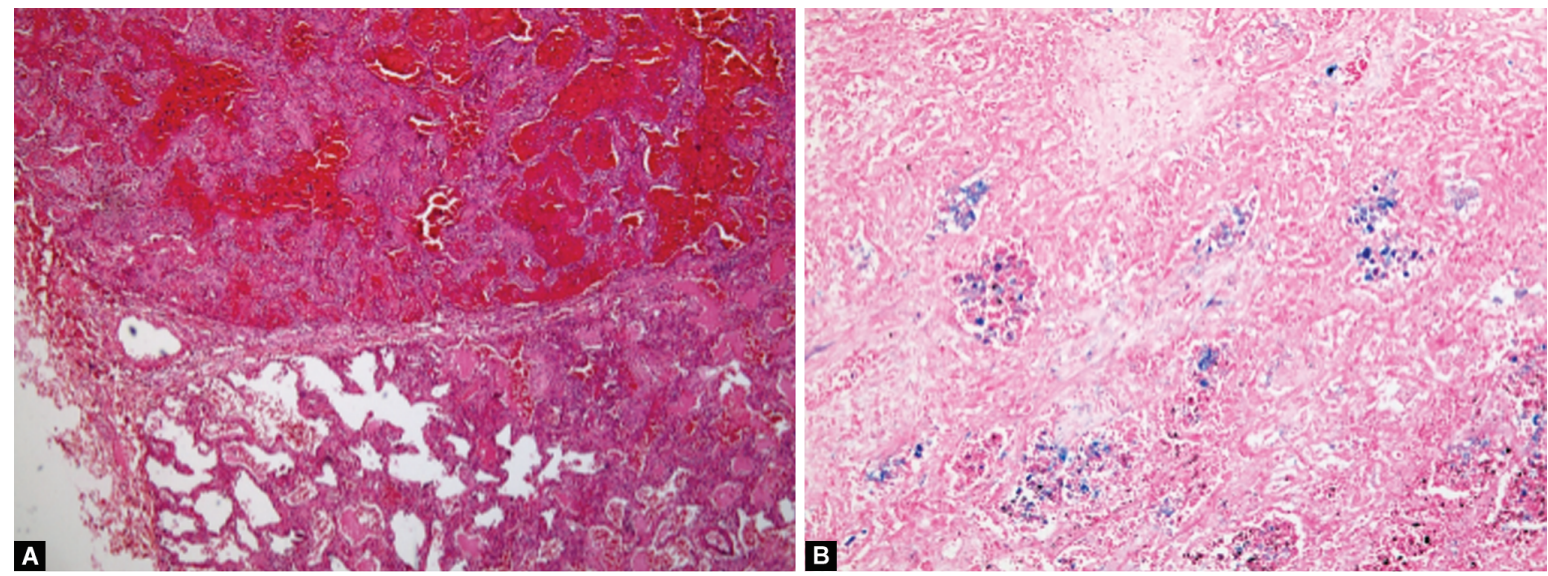

Sis.

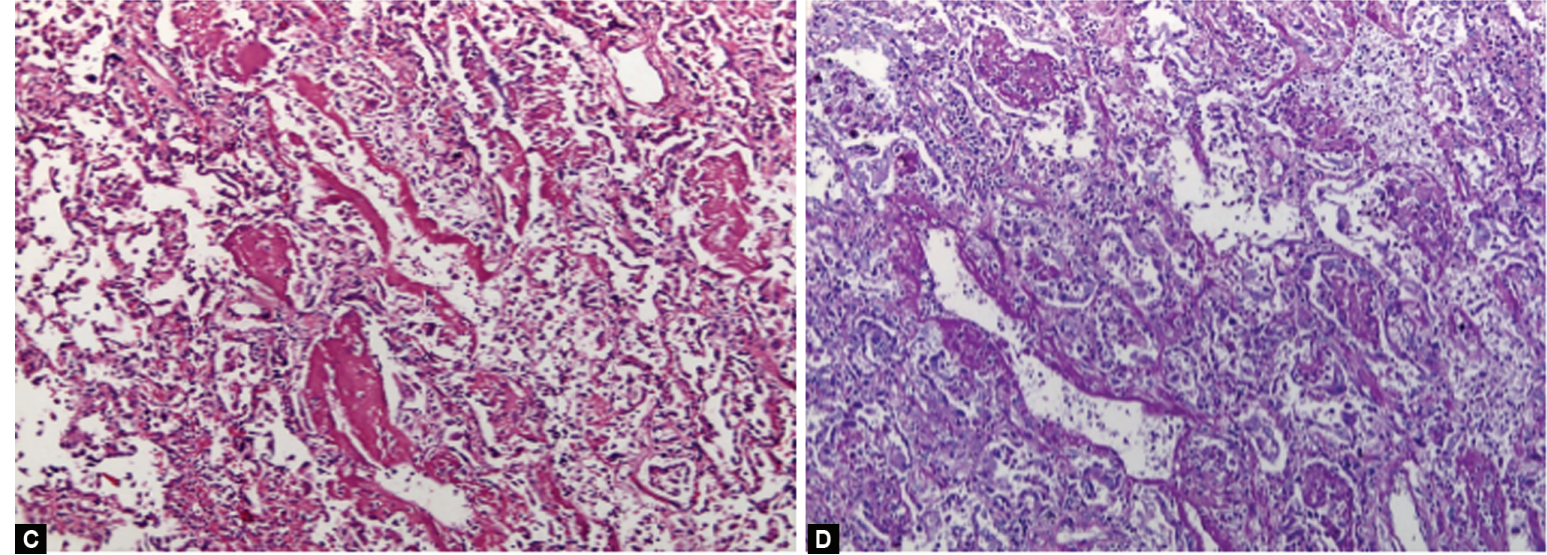

Figs 7A to D: (A) Photomicrograph from lung showing diffuse alveolar damage; (B) Perl's stain highlights hemosiderin-laden macrophages; (C and D) Deposition of hyaline membrane indicating diffuse alveolar damage [(A) H\&E stain; 40x, (B) Perl's stain; 100x, (C) H\&E stain; (D) PAS stain; 100x)
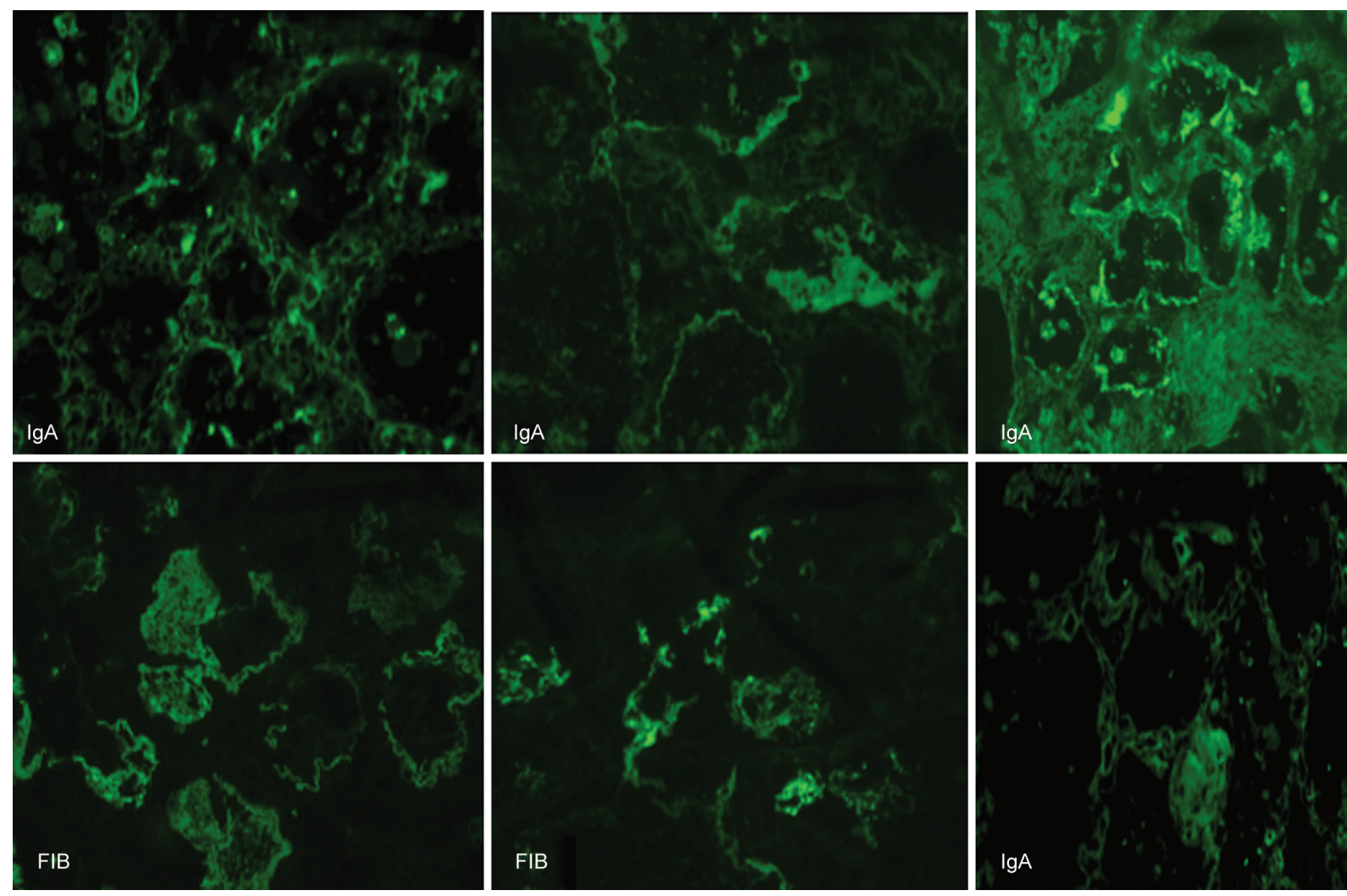

Fig. 8: Composite images of direct immunofluorescence done on lung tissue showing immune deposits of $\lg A$ and $\lg G$ with fibrinogen along the alveolar septal basement membrane and capillaries 

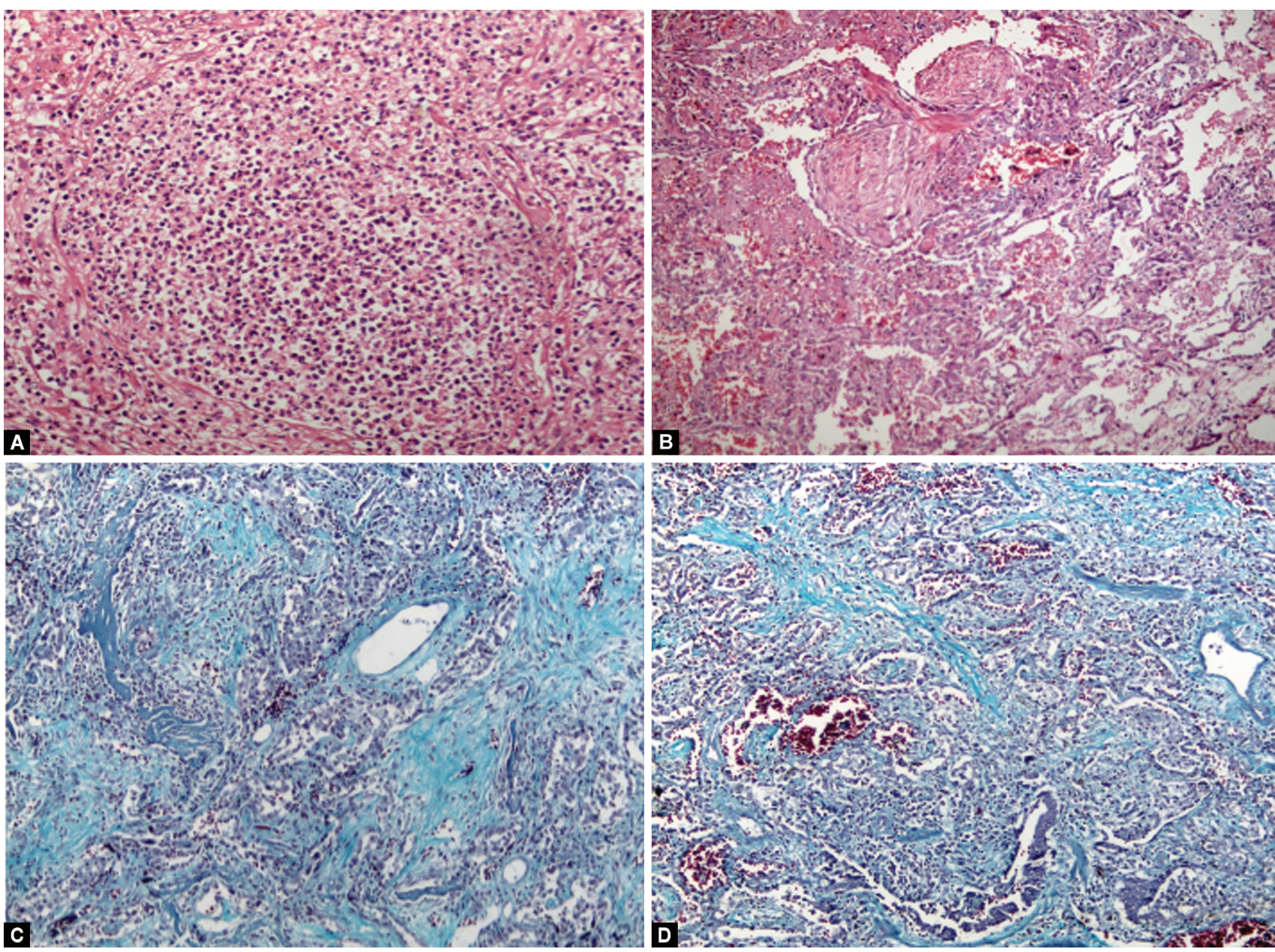

Figs 9A to D: (A) Photomicrograph from lung showing bronchopneumonia; (B to D) Lung showing obliterative bronchiolitis [(A and B) H\&E stain; (C and D) Masson trichrome stain; 100x]

18.5 years, and the male/female ratio was $16 / 3$. The most common presenting symptoms were dyspnea (84\%), hemoptysis (74\%), cough (53\%), and fever (47\%). ${ }^{3}$ Simultaneous involvement of kidneys and lungs was the most common (42\%), kidney involvement followed by lung involvement was seen in (37\%) cases, but alveolar hemorrhage precedes renal manifestations in $(21 \%)$ cases. The duration between diagnosis of IgAN and presentation of PRS is quite variable ranging from 7 days to 7 years. Those patients who underwent lung biopsy (8/11) showed capillaritis and three patients had positive IgA staining in lung biopsy. One case report of bronchiolitis obliterans was also reported in the case of IgA nephropathy. Common chest radiographic findings were consolidation/ground-glass opacities $(62.5 \%)$ and reticulonodular opacity $(25 \%)$.

This case highlights a rare manifestation of IgA nephropathy with the pulmonary-renal syndrome. The patient presented with pulmonary-renal syndrome only 3 months after the diagnosis of IgAN. IgA immune deposits in the lungs could be demonstrated due to the availability of tissue at autopsy which is otherwise not accessible in clinical practice.

\section{Conclusion}

This case highlights the fact that though pulmonary-renal syndrome is a very rare and fatal manifestation of IgA nephropathy, which is otherwise a slowly progressive disease. IgA-mediated pulmonary damage should be considered in the differential diagnosis of pulmonary hemorrhage with or without renal manifestations.

\section{References}

1. Rodriguez W, Hanania N, Guy E, et al. Pulmonary-renal syndromes in the intensive care unit. Crit Care Clin 2002;18(4):881-895. DOI: 10.1016/ s0749-0704(02)00029-5, x.

2. Rajagopala S, Parameswaran S, Ajmera JS, et al. Diffuse alveolar hemorrhage in IgA nephropathy: case series and systematic review of the literature. Int J Rheum Dis 2017;20(1):109-121. DOI: 10.1111/1756$185 X .12818$.

3. Hernández JL, Gómez-Román J, Rodrigo E, et al. Bronchiolitis obliterans and IgA nephropathy. A new cause of pulmonary-renal syndrome. Am J Respir Crit Care Med 1997;156(Pt 1):665-668. DOI: 10.1164/ajrccm.156.2.9610043. 